\title{
Corticolimbic structural alterations linked to health status and trait anxiety in functional neurological disorder
}

\author{
David L Perez ${ }^{1,2,3}$ Benjamin Williams, ${ }^{1}$ Nassim Matin, ${ }^{1}$ W Curt LaFrance, ${ }^{4}$ \\ Victor Costumero-Ramos, ${ }^{5,6}$ Gregory L Fricchione, ${ }^{7}$ Jorge Sepulcre, ${ }^{3}$ \\ Matcheri S Keshavan, ${ }^{8}$ Bradford C Dickerson ${ }^{3,9}$
}

- Additional material is published online only. To view please visit the journal online (http://dx.doi.org/10.1136/ jnnp-2017-316359)

For numbered affiliations see end of article.

\section{Correspondence to} Dr David L Perez, Massachusetts General Hospital, Departments of Neurology and Psychiatry 149 13th Street, Charlestown, MA 02129, USA; dlperez@ partners.org

DLP and BW contributed equally.

Received 2 May 2017 Revised 4 July 2017

Accepted 18 July 2017 Published Online First 26 August 2017

\section{ABSTRACT}

Objective Affective symptoms influence health status (health-related quality of life) in functional neurological disorder (FND), and the salience network is implicated in the pathophysiology of FND and mood/anxiety disorders. We hypothesised that self-reported health status and affective symptoms would map onto salience network regions and that patients with FND would show decreased insular volumes compared with controls. Methods This voxel-based morphometry study investigated volumetric differences in 26 patients with FND ( 21 women, 5 men; mean age $=40.3 \pm 11.5$ ) compared with 27 healthy controls ( 22 women, 5 men; mean age $=40.5 \pm 10.8$ ). Post hoc analyses stratified patients with FND by mental and physical health scores (Short Form Health Survey-36). Within-group analyses investigated associations with mental health, physical health, trait anxiety and depression in patients with FND. Results There were no volumetric differences between the complete FND cohort and controls. In stratified analyses, however, patients with FND reporting the most severe physical health impairments showed reduced left anterior insular volume compared with controls. In within-group analyses, impaired mental health and elevated trait anxiety were associated with increased right amygdalar volumes in patients with FND. The relationship between amygdalar volume and mental health, driven by emotional well-being deficits and role limitations due to emotional problems, was independent of sensorimotor functional neurological symptom severity and motor FND subtype. In secondary withingroup analyses, increased periaqueductal grey volume was associated with role limitations due to emotional problems. Impaired physical functioning correlated with decreased left anterior insular volumes.

Conclusions These findings support roles for several regions of the salience network in the pathophysiology of FND.

\section{INTRODUCTION}

CrossMark

To cite: Perez DL, Williams B, Matin N, et al. J Neurol Neurosurg Psychiatry 2017:88:1052-1059.
Patients with functional neurological disorder (FND)/conversion disorder report impairments in health-related quality of life (QoL), commonly referred to as health status. ${ }^{12}$ Functional neurological symptoms including abnormal movements, weakness, seizures, numbness, fatigue and pain frequently coexist with anxiety and depression. ${ }^{3} 4$ Psychosocial stressors and socioeconomic difficulties are also common in FND. ${ }^{5}$ Despite high morbidity and disease prevalence, ${ }^{6}$ many neurologists and psychiatrists are uncomfortable treating patients with FND. Furthermore, the limited understanding of the neurobiology of FND impedes the development of diagnostic and prognostic biomarkers.

Several studies suggest that health status in FND is partially independent of sensorimotor functional neurological symptoms and closely linked to affective symptoms. For example, a study of patients with psychogenic non-epileptic seizures (PNES) demonstrated that depression and somatic symptoms, but not seizure frequency, were significantly associated with reduced QoL. ${ }^{7}$ This finding has been independently replicated. ${ }^{8} 9$ A systematic review determined that affective symptoms, somatic complaints, dissociation, escape-avoidance coping, family dysfunction and other chronic psychiatric problems are linked to reported health status in PNES. ${ }^{1}$ Determinants of health status in other FND populations have been understudied. However, reports indicate that health impairments across FNDs are at least comparable with epilepsy, ${ }^{10}$ Parkinson's disease ${ }^{11}$ and lesion-induced weakness. ${ }^{2}$ Furthermore, negative expectation and negative attentional bias influence health perception, which may lead some patients with FND to overestimate their symptoms. ${ }^{12}$

Despite the use of health status as primary and secondary endpoints in FND clinical trials $^{1314}$ and growing interest in identifying prognostic biomarkers, few neuroimaging studies have examined brain-QoL relationships in neuropsychiatric disorders. Overall health is multidimensional and comprised of two main constructs-mental health and physical health. ${ }^{15}$ Considering the relationship between affective symptoms and health status in FND, neuroimaging studies probing emotion processing may provide insights into the neural correlates of subjective health perception. Studies of negative emotional processing in FND have identified heightened activity throughout the salience network, ${ }^{16-18}$ a distributed corticolimbic circuit involved in affective, cognitive and viscerosomatic functions that include the insula, dorsal anterior cingulate cortex (ACC), amygdala and periaqueductal grey as core components. ${ }^{19-21}$ 
Functional MRI (fMRI) studies report increased amygdalar activity during the viewing of affectively valenced faces ${ }^{22} 23$ and heightened coupling of amygdalar-supplementary motor area activity 2224 in FND compared with controls. Increases in insular, dorsal ACC and periaqueductal grey activations have also been identified in FND. ${ }^{23} 2627$

Structural MRI studies further support involvement of salience network-related corticolimbic brain areas in the neuropathophysiology of FND. Decreased grey matter volume in the ACC was observed in patients with PNES compared with controls. ${ }^{28}$ Insular structural alterations have been reported in PNES, ${ }^{29}$ and patients with a somatic symptom disorder with predominant pain exhibited decreased cingulo-insular volumes compared with controls. ${ }^{30}$ Neuroimaging meta-analyses of FND $^{31}$ and other somatic symptom disorders ${ }^{32}$ support a neurobiological role for multiple regions within the salience network. Recently, we reported that functional neurological symptom severity was associated with reduced left anterior insular volume in women with FND, while trauma-related hyperarousal correlated with decreased dorsal ACC grey matter. ${ }^{33}$ These findings demonstrated the utility of within-group designs to dimensionally characterise brain-symptom relationships in FND.

In this voxel-based morphometry (VBM) MRI study, wholebrain comparative analyses were performed in 26 patients with FND and 27 healthy subjects. Post hoc analyses stratified patients with FND by mental and physical health scores to further study group-level differences. In addition, we used a within-group dimensional approach to investigate grey matter volumetric associations with reported mental health, physical health, trait anxiety and depression in patients with FND. Considering the literature characterising alterations within the salience network in FND, as well as the recognised relationship between poor health status and affective symptoms, we hypothesised that self-reported measures of health status and affective symptoms would both map onto salience network brain areas. Building on our prior observation that sensorimotor functional neurological symptom severity correlated with reduced insular volume, ${ }^{33}$ we also hypothesised that reported physical health impairments would be associated with reduced left anterior insular grey matter and that patients with FND compared with controls would show decreased insular volume.

\section{METHODS}

Methods were adapted from those detailed in ref 33.

\section{Participants and psychometric measures}

We recruited 26 FND subjects (21 women, 5 men; mean age $=40.3 \pm 11.5$; range $22-60$; average illness duration $=3.6 \pm 4.3$ years) from the Functional Neurological Disorders Clinic at the Massachusetts General Hospital. ${ }^{34}{ }^{35}$ Diagnoses were based on clinical evaluation by a dual-trained and board-certified neurologist and psychiatrist (DLP). Twenty-seven healthy controls ( 22 women, 5 men; mean age $=40.5 \pm 10.8$; range $21-60$ ) were recruited through local advertisements. Patients met diagnostic criteria for clinically established functional movement disorders $(n=13),{ }^{36}$ documented $(n=9)$ or clinically established PNES $(\mathrm{n}=1)^{37}$ and/or exhibited positive signs on examination for functional weakness $(n=12) . .^{38}$ Nine patients had mixed motor FND, and a distinct nine subjects exhibited non-dermatomal sensory deficits. Exclusion criteria included any major neurological disorder with MRI abnormalities, epilepsy, poorly controlled medical illnesses with known central nervous system consequences, ongoing substance dependence, a history of mania or psychosis and/or active suicidality. Psychiatric comorbidities for all subjects were assessed through the Structured Clinical Interview (SCID-I) for DSM-IV-TR. Twenty-four of 26 patients with FND met criteria for current comorbid psychiatric diagnoses, and 20 were on psychotropic medications. All healthy subjects were without psychiatric comorbidities as evaluated by the SCID-I, and were not on any psychotropic medications. Clinical information is detailed in online supplementary table 1 . All subjects signed informed consent and the Partners Human Research Committee approved this study.

As part of a detailed psychometric battery, subjects completed the Short Form Health Survey-36 (SF-36) ${ }^{39}$ the Spielberger Trait Anxiety Inventory (STAI-T ${ }^{40}$ and the Beck Depression Inventory-II (BDI). ${ }^{41}$ The SF-36 is a widely used and validated 36-item self-report questionnaire assessing health status (also known as health-related QoL) and consists of two composite categories: physical health and mental health. The composite mental health score, which characterises overall emotional wellness and the extent to which emotional problems impact an individual's daily functioning, is comprised of four subscales including emotional well-being (five items), energy/fatigue (four items), role limitations due to emotional problems (three items) and social functioning (two items). The composite physical health score, which quantifies overall physical wellness and the extent to which physical symptoms limit an individual's abilities to work and/ or perform regular daily activities, is also comprised of four subscales including physical functioning (10 items), general health (five items), role limitations due to physical health (four items) and pain (two items). For the remainder of this article, the mental health composite score is referred to as 'mental health' and the physical health composite score is referred to as 'physical health'. The SF-36 subscales are referred to by their specific subdomains, as outlined above. The STAI-T is a 20 -item self-report measure of trait anxiety, and the BDI is a 21 -item self-report measure for depression.

Other psychometric measures included the Patient Health Questionnaire-15 (PHQ-15) ${ }^{42}$ and the Conversion Disorder subscale of the Screening for Somatoform Symptoms-7 scale (SOMS:CD), ${ }^{43}$ which were only used in this study to control for functional neurological symptom severity (see below).

\section{MRI data acquisition}

Subjects were placed in a Siemens 3 Tesla Trio scanner to acquire a 3D T1-weighted magnetisation prepared rapid acquisition gradient echo sequence with the following parameters: orientation $=$ sagittal; matrix size $=256 \times 256$; voxel size $=1 \times 1 \times 1 \mathrm{~mm}$; slice thickness $=1 \mathrm{~mm}$, slices $=160$; repetition time $=2300 \mathrm{~ms}$; echo time $=2.98 \mathrm{~ms}$; field of view $=256 \mathrm{~mm}$. Bitemporal foam pads were used to restrict head motion.

\section{MRI data preprocessing}

Statistical Parametric Mapping 8 (SPM8; www.fil.ion.ucl.ac.uk/ $\mathrm{spm} /$ ) and the VBM8 toolbox were used to analyse data. Scans were inspected for quality and reoriented along the anterior-to-posterior commissure. Images were segmented into grey matter, white matter and cerebrospinal fluid components. The diffeomorphic anatomical registration through exponentiated lie algebra (DARTEL) was applied to the grey matter images for spatial normalisation. Non-linear modulation using the Jacobian determinants derived from the normalisation process was implemented to control for individual whole-brain volume differences. The modulated grey matter images were smoothed with an $8 \mathrm{~mm}$ full-width-at-half-maximum Gaussian kernel. 


\section{MRI statistical analysis}

After preprocessing, all between-group comparisons (eg, 26 FND vs 27 healthy controls) were performed using the general linear model in SPM adjusting for age and gender. For stratified post hoc analyses, patients with FND in the top 50 percentile of impaired physical health $(n=13)$ or mental health $(n=13)$ were compared with controls. For within-group analyses in patients with FND, SPM-based multiple regression was performed to examine relationships between covariates-of-interest and grey matter volumes with age and gender included as nuisance variables. Separate regression analyses were implemented for overall health status (SF-36 total score), mental health, physical health, STAI-T and BDI scores. Given that the individual SF-36 subscales have greater symptom specificity, secondary analyses were also performed using the eight subscales as covariates-of-interest. For all analyses, SF-36 scores were inverted so that higher scores reflected worse health status.

Whole-brain corrections for multiple comparisons at the peak voxel-level used a family-wise error (FWE) rate of $p<0.05$. Given that a priori hypotheses related to salience network brain areas as proposed by Seeley et $a l^{19}$ and Bickart et $a l^{21}$, we defined bilateral regions-of-interest in the insula, ACC and amygdala using the WFU Pickatlas to perform small volume corrections (SVCs). Since the WFU Pickatlas does not include the periaqueductal grey, SVC for this area was performed using a $12 \mathrm{~mm}$ sphere centred at MNI coordinate $(1,-29,-12)$ based on an activation likelihood estimation meta-analysis. ${ }^{20}{ }^{44}$ An FWE rate of $\mathrm{p}<0.05$ was used for SVCs at the peak-voxel level.

In post hoc within-group analyses, health status-related volumetric findings were controlled for PHQ-15 and SOMS:CD scores in separate regression analyses to test if observed relationships were independent of sensorimotor functional neurological symptom severity. Additional regression analyses were also performed on all significant within-group findings to control separately for motor FND subtypes.

\section{RESULTS \\ Associations between health status and affective symptoms in FND}

See online supplementary figure 1 for a description of the relationships between psychometric variables of interest. See online supplementary table 2 for clinical scores.

\section{Volumetric differences in patients with FND compared with controls}

Compared with healthy controls, patients with FND did not show any whole-brain corrected or SVC group-level differences.

In stratified post hoc analyses, however, FND patients with the greatest reported physical health impairments showed decreased left anterior insular grey matter volumes $\left(\mathrm{p}_{\mathrm{svc}}=0.007\right)$ compared with controls. Patients with FND reporting the most severe mental health impairments displayed increased right posterior-lateral cerebellar grey matter volumes $\left(\mathrm{p}_{\text {whole-brain-cor- }}\right.$ rected $=0.036$ ) compared with controls. There were no other statistically significant between-group findings (see figure 1, table 1 , online supplementary figure 2 and supplementary table 3 ).

Within-group grey matter associations with health status and affective symptoms

In 26 patients with FND, increased right amygdalar grey matter volume was associated with reduced mental health $\left(\mathrm{p}_{\text {small-vol- }}\right.$ ume-corrected(svc) $=0.004)$ and elevated trait anxiety $\left(\mathrm{p}_{\mathrm{svc}}=0.018\right)$ scores in separate SPM regression analyses (see figure 2).
Insular and Cerebellar Volumetric Alterations in Patients with FND Reporting Markedly Impaired Physical and Mental Health Compared to Controls

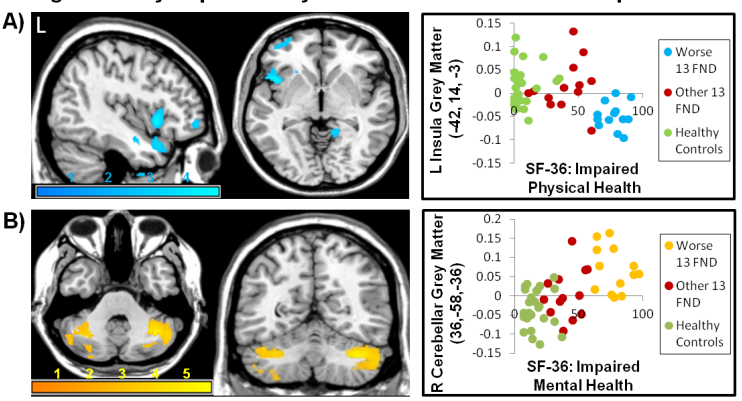

Figure 1 Patients with functional neurological disorder (FND) reporting markedly impaired physical and mental health showed decreased insular and increased cerebellar volumes compared to controls. (A) Compared with healthy controls, patients with FND in the top-half of individuals reporting markedly impaired physical health $(N=13)$ exhibited decreased left anterior insular volume $\left(p_{\text {svu }}=0.007\right.$, peak $z$-score $\left.=-4.25\right)$.

(B) Individuals with FND in the top-half of those reporting impaired mental health displayed increased right posterior-lateral cerebellar grey matter volumes $\left(p_{\text {whole-brain corrected }}=0.036\right.$, peak $z$-score $\left.=4.80\right)$ compared with controls. Note: physical and mental health are based on the two composite categories of the Short Form Health Survey-36 (SF-36) scores. All SF-36 scores were inverted so that higher scores represent worse health status. To display in the scatter plots an age and gender-adjusted measure of grey matter volumes for visualisation purposes only, the MarsBar toolbox (http://marsbar.sourceforge.net/)was used to extract mean grey matter volumes for each subject at a $5 \mathrm{~mm}$ sphere centred at the peak voxel of statistically significant findings.

The association between mental health deficits and increased right amygdalar volumes held controlling for PHQ-15 $\left(\mathrm{p}_{\mathrm{svc}}=0.007\right)$ and SOMS:CD $\left(\mathrm{p}_{\text {svc }}=0.006\right)$ scores. Only trend level associations were appreciated between physical health impairments and decreased left anterior insular volumes $\left(\mathrm{p}_{\text {uncorrected }}<0.001\right)$.

In separate secondary within-group analyses performed using the eight SF-36 subscales, increased right amygdalar grey matter volume positively correlated with role limitations due to emotional problems $\left(\mathrm{p}_{\mathrm{svc}}<0.001\right)$ and reduced emotional well-being $\left(\mathrm{p}_{\mathrm{svc}}=0.014\right)$ subscores (see figure 3 and online supplementary figure 3 ). These findings held when controlling for PHQ-15 ( $\mathrm{p}_{\mathrm{svc}}=0.001$; $\left.\mathrm{p}_{\mathrm{svc}}=0.022\right)$ and SOMS:CD $\left(\mathrm{p}_{\mathrm{svc}}=0.001 ; \mathrm{p}_{\mathrm{svc}}=0.016\right)$ scores. Elevated role limitations due to emotional problems subscale scores were also associated with increased left amygdalar $\left(\mathrm{p}_{\text {svc }}=0.030\right)$ and periaqueductal grey $\left(\mathrm{p}_{\text {svc }}=0.003\right)$ volumes, which remained significant adjusting for PHQ-15 $\left(\mathrm{p}_{\mathrm{svc}}=0.033 ; \mathrm{p}_{\mathrm{svc}}=0.001\right)$ and SOMS:CD $\left(\mathrm{p}_{\mathrm{svc}}=0.031 ; \mathrm{p}_{\mathrm{svc}}=0.004\right)$ scores. Impaired physical functioning subscale scores correlated with decreased left anterior insular volume $\left(\mathrm{p}_{\mathrm{syc}}=0.039\right)$, although this finding did not hold when controlling for functional neurological symptom severity.

In post hoc within-group analyses, all statistically significant findings held controlling for motor FND subtypes, except for the association between left anterior insular volumes and physical functioning subscale scores, which did not remain significant when controlling for any motor subtype. There were no statistically significant within-group associations for BDI scores. See online supplementary tables 4-6 for complete findings.

\section{Discussion}

This structural MRI study supports involvement of corticolimbic components of the salience network in the neurobiology of FND. While there were no statistically significant group-level 
Table 1 Statistically significant between-group and within-group grey matter volumetric associations in patients with functional neurological disorder and healthy controls

\begin{tabular}{|c|c|c|c|c|c|c|c|c|}
\hline \multirow[b]{2}{*}{ Analysis } & \multirow[b]{2}{*}{ Measures of interest } & \multirow{2}{*}{$\begin{array}{l}\text { Cerebral regions } \\
\text { (Brodmann area) }\end{array}$} & \multicolumn{3}{|c|}{$\begin{array}{l}\text { Peak coordinates in MNI } \\
\text { Space }(\mathrm{mm})\end{array}$} & \multirow{2}{*}{$\begin{array}{l}\text { Peak voxel } \\
\text { Z-score }\end{array}$} & \multirow{2}{*}{$\begin{array}{l}\text { p Value } \\
\text { (SVC) }\end{array}$} & \multirow{2}{*}{$\begin{array}{l}\text { Cluster extent } \\
\left(\mathrm{mm}^{3}\right)\end{array}$} \\
\hline & & & $\mathrm{x}$ & y & z & & & \\
\hline \multirow[t]{2}{*}{ Between group } & $\begin{array}{l}\text { Poor physical health } \\
13 \mathrm{FND}<27 \mathrm{HC}\end{array}$ & L anterior insula (48) & -42 & 14 & -3 & -4.25 & $<0.001(0.007)$ & 342 \\
\hline & $\begin{array}{l}\text { Poor mental health } \\
13 \mathrm{FND}>27 \mathrm{HC}\end{array}$ & R cerebellum, crus I & 36 & -58 & -36 & 4.80 & $<0.001\left(0.036^{*}\right)$ & 1453 \\
\hline \multirow[t]{7}{*}{ Within group } & $\begin{array}{l}\text { Impaired mental health } \\
\text { positive associations }\end{array}$ & R amygdala (34) & 28 & -1 & -18 & 3.90 & $<0.001(0.004)$ & 415 \\
\hline & $\begin{array}{l}\text { STAI-Trait anxiety } \\
\text { positive associations }\end{array}$ & R amygdala (34) & 21 & -1 & -17 & 3.46 & $<0.001(0.018)$ & 132 \\
\hline & $\begin{array}{l}\text { Impaired emotional well-being } \\
\text { positive associations }\end{array}$ & R amygdala (34) & 27 & -1 & -18 & 3.54 & $<0.001(0.014)$ & 170 \\
\hline & \multirow{3}{*}{$\begin{array}{l}\text { Role limitations due to emotional } \\
\text { problems } \\
\text { positive associations }\end{array}$} & R amygdala (20) & 30 & -3 & -20 & 4.56 & $<0.001(<0.001)$ & 1018 \\
\hline & & Periaqueductal grey & -3 & -37 & -12 & 4.12 & $<0.001(0.003)$ & 534 \\
\hline & & $\begin{array}{l}\text { L amygdala/ } \\
\text { anterior hippocampus } \\
(20)\end{array}$ & -27 & -6 & -18 & 3.29 & $<0.001(0.030)$ & 596 \\
\hline & $\begin{array}{l}\text { Impaired physical functioning } \\
\text { negative associations }\end{array}$ & $\mathrm{L}$ anterior insula (48) & -39 & 8 & -11 & -3.78 & $<0.001(0.039)$ & 179 \\
\hline
\end{tabular}

Note: Physical and mental health are based on the two composite categories of the Short Form Health Survey-36 (SF-36). The 'emotional well-being', 'role limitations due to emotional problems', and 'physical functioning' measures are individual subscales of the SF-36. All SF-36 scores were inverted so that higher scores represent worse health status.

*Denotes whole-brain corrected $\mathrm{p}$ value.

FND, functional neurological disorder; HC, healthy controls; L, left; MNI, Montreal Neurological Institute; R, right; STAI-Trait Anxiety, Spielberger Trait Anxiety Inventory; SVC, small volume corrected.

differences between patients with FND and controls, stratified post hoc analyses showed that the subset of FND subjects with the most severely impaired physical health showed reduced left anterior insular grey matter volumes compared with controls. In addition, FND patients with the most severely impaired mental health exhibited increased posterior-lateral cerebellar grey matter volumes compared with controls. In complementary within-group analyses performed in patients with FND, impaired reported mental health and increased trait anxiety were each associated with increased right amygdalar grey matter volumes. The association between amygdalar volume and mental health, driven by deficits in emotional well-being and role limitations due to emotional problems, was independent of sensorimotor functional neurological symptom severity and motor subtype. In secondary within-group analyses, role limitations due to emotional problems subscale scores were also associated with increased periaqueductal grey volume, and impaired physical functioning subscale scores correlated with reduced left anterior insular grey matter volumes.

In this study, we identified both between-group and within-group associations with left anterior insular volume. Compared with healthy controls, the subset of patients with FND reporting the greatest deficits in physical health demonstrated reduced left anterior insular volume. In FND subjects, impaired physical functioning subscale scores also correlated with decreased left anterior insular volume. These inter-related findings are consistent with and extend our prior report of inverse correlations between functional neurological symptom severity and anterior insular volume in FND. ${ }^{33}$ As a paralimbic, multimodal brain area implicated in the processing and integration of viscerosomatic, interoceptive, affective, cognitive and visceromotor functions and a core region of the salience network, we have postulated an important role for the insula in the pathophysiology of FND. ${ }^{1745} 46$ The anterior insula has been linked specifically to emotional and self-awareness ${ }^{47}$ and modulates the balance between frontoparietal control and default mode networks. ${ }^{48}$ We have developed the construct of 'neural functional unawareness' as a model framework to potentially explain the failed integration of affective, cognitive and sensorimotor functions in FND. ${ }^{1746}$ Given the multiplicity of symptoms and variable levels of physical disability seen in FND, our stratified group-level analyses suggest that biomarkers for FND may be particularly robust in patients with high physical symptom burden. The stratified group-level observations are notable given that quantitative structural differences in motor FND populations compared with healthy subjects have been inconsistent, ${ }^{28294950}$ with one study reporting no cortical differences in 15 individuals with functional weakness compared with healthy controls. ${ }^{50}$

The link between reduced mental health, increased trait anxiety and amygdalar volume in patients with FND may be contextualised through neuroimaging, neuroendocrine and autonomic studies. As previously noted, fMRI studies have consistently identified increased amygdalar activity during affective processing in FND, ${ }^{16222325}$ and heightened amygdalar activity has been recently linked to negative attentional bias in individuals with pathological health anxiety. ${ }^{51}$ In healthy subjects, individual differences in trait anxiety mediate amygdalar activity during unconscious emotional processing, ${ }^{52}$ and trait-level differences in fearfulness, ${ }^{53}$ anxiety ${ }^{54}$ and negative affect ${ }^{55}$ correlate positively with amygdalar volumes. Trait anxiety, a risk factor for the development of psychopathology, is also linked to reduced parasympathetic tone ${ }^{56}$ and increased cortisol release. ${ }^{57}$ Studies examining hypothalamic-pituitary-adrenal (HPA) axis function in FND, particularly those with PNES, show cortisol abnormalities. For example, patients with PNES exhibit increased diurnal cortisol levels compared with controls, ${ }^{58}$ and attentional bias for threatening stimuli correlates with salivary cortisol levels 


\section{Grey Matter Relationships with Health Status and Trait Anxiety in Functional Neurological Disorder}
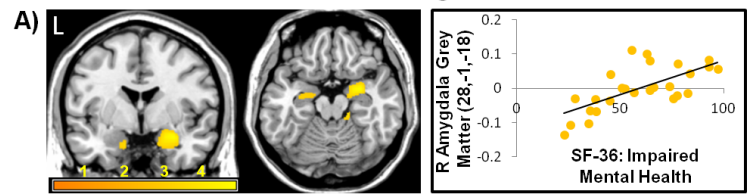

B)
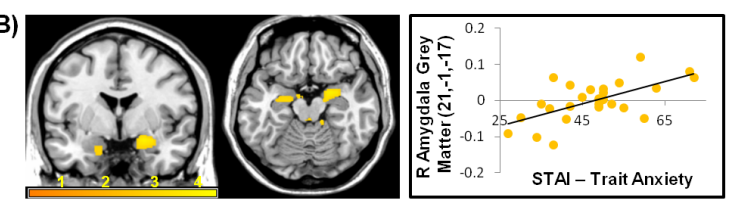

C)
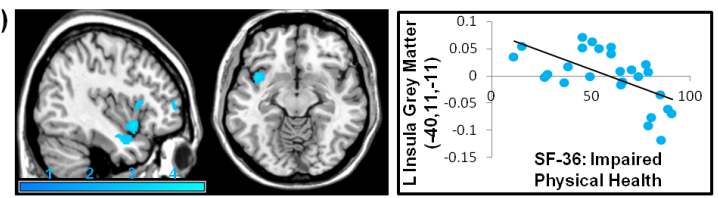

Figure 2 Individual differences in mental health and trait anxiety correlated with amygdalar volumes in functional neurological disorder. (A) Impaired mental health was associated with increased right amygdalar volumes $\left(p_{\text {svc }}=0.004\right.$; peak $z$-score $\left.=3.90\right)$. (B) Heightened trait anxiety, as measured by the Spielberger Trait Anxiety Inventory (STAI-Trait Anxiety), correlated with increased right amygdalar volumes $\left(p_{s u}=0.018\right.$; peak z-score=3.46). (C) Impairments in physical health showed only a trendlevel association with decreased left anterior insular grey matter volumes $\left(p_{\text {uncorrected }}<0.001\right.$; peak z-score $\left.=-3.51\right)$. Note: mental and physical health are based on the two composite categories of the Short Form Health Survey-36 (SF-36) scores. All SF-36 scores were inverted so that higher scores represent worse health status. Images are thresholded at a voxelwise uncorrected $p$ value of 0.005 . To display in the scatter plots age and gender-adjusted measures of grey matter volumes for visualisation purposes only, the MarsBar toolbox (http://marsbar.sourceforge.net/) was used to extract mean grey matter volumes for each subject at a $5 \mathrm{~mm}$ sphere centred at the peak coordinate of statistically significant findings.

in this population. ${ }^{59}$ Autonomic studies examining heart rate and heart rate variability have characterised increased sympathetic and decreased parasympathetic tone in motor FND. ${ }^{60-62}$ Patients with functional movement disorders, for example, show decreased vagal tone as measured by increased heart rate and reduced variability. ${ }^{62}$ Given the convergence of neuroendocrine and autonomic findings in FND, as well as the heterogeneity of amygdalar volumes in our FND cohort (see online supplementary figure 2), future studies should examine relationships between amygdalar volume, HPA axis function, autonomic profiles and selective serotonin reuptake inhibitor response in FND.

In secondary analyses, we observed a relationship between role limitations due to emotional problems subscale scores and increased periaqueductal grey volume, a region implicated in fear, anxiety, pain modulation, homeostatic functions and defensive behaviours. ${ }^{206364}$ In the periaqueductal grey, ventrolateral and dorsolateral segments are implicated in freezing and fightor-flight responses respectively. Aybek and colleagues have characterised heightened periaqueductal grey responses to affectively valenced faces in patients with FND compared with controls and posited links between abnormal threat responses and functional motor symptoms. ${ }^{23}$ The periaqueductal grey has also been connected to the neurobiology of panic disorder, ${ }^{65}$ and there is considerable overlap between panic attacks and paroxysmal FND symptoms. ${ }^{66}$ Additionally, increases in periaqueductal grey volume have been reported in primary dysmenorrhea ${ }^{67}$ and
Regional Salience Network Grey Matter Relationships with Health Status Subscores in Functional Neurological Disorder
A)

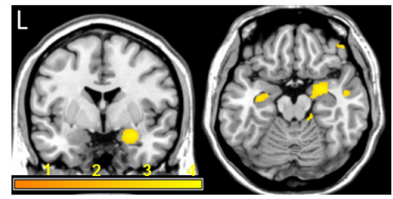

B)

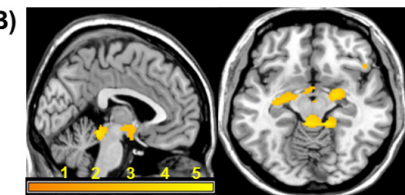

C)

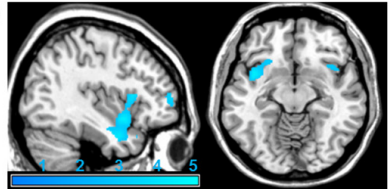

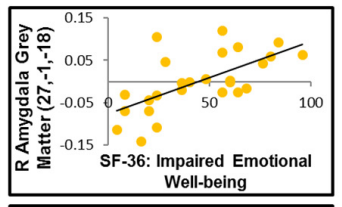
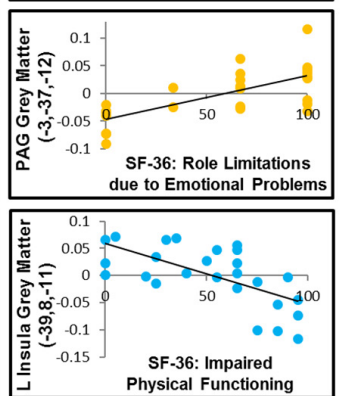

Figure 3 Individual differences in health status subscores linked to regional salience network grey matter volumes in functional neurological disorder. (A) Impaired emotional well-being, as measured by the Short Form Health Survey-36 (SF-36), correlated with increased right amygdalar grey matter volumes ( $p_{\text {svc }}=0.014$, peak $z$-score=3.54). (B) Role limitations due to emotional functioning subscale scores were associated with increased bilateral amygdalar (see online supplementary figure 3) and periaqueductal grey (PAG) volumes $\left(p_{\text {svc }}=0.003\right.$, peak $z$-score $\left.=4.12\right)$. $(C)$ Impaired physical functioning subscale scores correlated with decreased left anterior insular grey matter volumes $\left(p_{\text {svc }}=0.039\right.$, peak $z$-score $\left.=-3.78\right)$. Note: SF-36 scores were inverted so that higher scores represent worse health status. The 'emotional well-being' subscale is a five-question measure where subjects rate how they have been feeling emotionally over the past 4 weeks, with each question rated on a 6-point Likert scale. The 'role limitations due to emotional problems' subscale is a three-item measure where subjects rate whether emotional problems limited their ability to work or perform daily activities over the past 4 weeks; questions are dichotomously scored as 'yes' or 'no'. The 'physical functioning' subscale is a 10-item measure where subjects rate on a 3-point Likert scale to what extent their health impedes their daily activities (i.e. climbing a flight of stairs). Images are thresholded at a voxel-wise uncorrected $p$ value of 0.005 . To display in the scatter plots age and gender-adjusted measures of grey matter volumes for visualisation purposes only, the MarsBar toolbox (http://marsbar.sourceforge.net/) was used to extract mean grey matter volumes for each subject at a $5 \mathrm{~mm}$ sphere centred at the peak coordinate of statistically significant findings.

migraine headaches, ${ }^{44}$ conditions frequently comorbid in FND. Given the phenotypic heterogeneity in FND, we speculate that abnormally coordinated ventrolateral and dorsolateral periaqueductal grey activity, as well as altered interactions between the periaqueductal grey and its regulatory limbic/paralimbic regions, may play a role in the generation of specific functional neurological motor manifestations.

Associations between self-reported health status and regional brain volumes, while potentially related to illness severity, may alternatively reflect perceived levels of physical and emotional limitations driven by negative expectations and negative attentional bias. ${ }^{45}$ Arthur Barsky and colleagues proposed that somatosensory amplification, involving critical nodes of the salience network, mediates the maladaptive tendency to experience benign bodily sensations as noxious, intense and intrusive. ${ }^{68}$ In support of this interpretation, one study demonstrated that patients with functional tremor overestimate the frequency of their symptoms in comparison with objectively obtained 
actigraphy data. ${ }^{12}$ Patients with functional movement disorders also show exaggerated startle responses to both positively and negatively valenced stimuli, supporting a negative attentional bias in this population. ${ }^{69}$ Nocebo hyperalgesia has been linked to decreased opioid and dopaminergic activity in the insula and periaqueductal grey, ${ }^{70}$ and negative attentional bias has been linked to increased amygdalar activity in somatic symptom disorders. ${ }^{51}$ Future studies investigating health status-related biomarkers in FND should collect both self-reported and objective markers of physical and affective symptoms to further clarify the relationships identified in this study.

While beyond a priori regions-of-interest, we also observed increased right posterior-lateral cerebellar (crus I) grey matter volumes in the subset of patients with FND reporting the most severe deficits in mental health compared with controls. The increased cerebellar volume observed in our FND cohort maps onto areas implicated in executive and affective functions, ${ }^{71}$ and resting state functional connectivity analyses in 1000 healthy subjects have linked posterior-lateral aspects of the cerebellum to the salience network. ${ }^{72}$ Prior studies have identified structural and functional cerebellar abnormalities in $\mathrm{FND}^{24} 28$ 73-75 and other somatic symptom disorders. ${ }^{7677}$ These findings are noteworthy given the increasingly recognised role of the cerebellum in the modulation of cognitive and affective functions around a homeostatic baseline, termed the universal cerebellar transform. ${ }^{78}$ Across a number of systems-level investigations, the posterior-lateral lobule of the cerebellum (including crus I) and the midline vermis are implicated in negative emotional processing and aversive responses. For example, in a taskbased fMRI study performed in health subjects, the posterior cerebellum displayed increased activations to noxious thermal stimuli and showed heightened functional connectivity to the anterior insula, dorsal ACC and periaqueductal grey. ${ }^{79}$ Future research should continue to investigate the potential role of cortical-subcortical-cerebellar interactions in the pathophysiology of FND.

Limitations of this study include psychotropic medication use in patients, modest sample size, reliance on self-report measures and the mixed symptomatology of our FND cohort. Although still debated in the field, ${ }^{80}$ we have previously outlined the utility of studying patients across the motor FND spectrum using a transdiagnostic approach. ${ }^{33} 34{ }^{46}$ In 100 consecutive patients with FND evaluated in our clinic, 1 in 4 showed mixed functional motor symptoms at initial evaluation, reflecting the inherent overlap across populations. ${ }^{35}$ Furthermore, a strength of this study is the ability to control for motor FND subtypes in within-group analyses. The reliance on self-report measures constitutes a potential limitation due to the possible mismatch between self-reported and objective impairments. However, for measures of health-related QoL, self-report is the gold standard, and capturing subjects' personal views of their mental and physical health is one of the specific aims of this study. Additionally, we did not exclude FND patients with a high affective symptom burden. Comorbid psychiatric conditions are exceedingly common in $\mathrm{FND},{ }^{2}$ and the inclusion of individuals with psychiatric comorbidities increases the external validity of our cohort to clinical practice. We did not identify any within-group associations with depression scores, suggesting that depression may be particularly multifactorial in FND. Future neuroimaging studies should investigate shared biological underpinnings of FND and personality disorders, as well as potential differences in personality profiles and developmental trajectories across the spectrum of motor FND. ${ }^{81}{ }^{82}$ Lastly, additional research is needed to clarify the significance of lateralised amygdalar, insular and cerebellar volumetric findings, particularly their relationship to autonomic function and conscious versus unconscious affective processing. ${ }^{83}$

In conclusion, group-level reductions in left anterior insular volume and increases in right posterior-lateral cerebellar volume were observed in patients with FND reporting the most severe deficits in physical and mental health, respectively. This study also identified associations between trait anxiety, mental health and individual differences in amygdalar volumes in FND. Given the heterogeneity inherent to FND, we demonstrate the utility of investigating individual differences and stratifying by symptom severity. This MRI study is also one of the first to probe brainQoL relationships in neuropsychiatric disorders and suggests that mental and physical health perception may have at least partially distinct neural mechanisms. Our findings strengthen support for involvement of several regions of the salience network in the pathophysiology of FND.

\section{Author affiliations}

'Department of Neurology, Functional Neurology Research Group, Cognitive Behavioral Neurology Unit, Massachusetts General Hospital, Harvard Medical School, Boston, Massachusetts, USA

${ }^{2}$ Department of Psychiatry, Neuropsychiatry Unit, Massachusetts General Hospital, Boston, Massachusetts, USA

${ }^{3}$ Athinoula A. Martinos Center for Biomedical Imaging, Massachusetts General Hospital, Charlestown, Massachusetts, USA

${ }^{4}$ Neuropsychiatry and Behavioral Neurology Division, Departments of Psychiatry and Neurology, Rhode Island Hospital, Brown University, Alpert Medical School, Providence, Rhode Island, USA

${ }^{5}$ Department of Methodology, University of Valencia, Valencia, Spain

${ }^{6}$ Department of Basic and Clinical Psychology and Psychobiology, University Jaume I, Castellón de la Plana, Spain

${ }^{7}$ Department of Psychiatry, Benson-Henry Institute for Mind Body Medicine, Massachusetts General Hospital, Boston, Massachusetts, USA

${ }^{8}$ Department of Psychiatry, Beth Israel Deaconess Medical Center, Harvard Medical School, Boston, Massachusetts, USA

${ }^{9}$ Department of Neurology, Frontotemporal Disorders Unit, Massachusetts General Hospital, Boston, Massachusetts, USA

Contributors DLP, WCL, MSK and BCD designed the study. DLP, BW and NM collected the data. DLP, BW, NM and VC-R performed the analyses. DLP and BW drafted the manuscript. All authors critically interpreted the results and critically revised the manuscript.

Funding DLP was funded by the Dupont Warren and Livingston Fellowships, Massachusetts General Hospital Physician-Scientist Development Award, and the Sidney R. Baer Jr. Foundation.

Competing interests $B C D$ : consultant at Merck, Med Learning Group and Haymarket; royalties from Oxford University Press and Cambridge University Press; on the editorial board of Neuroimage: Clinical, Cortex, Hippocampus, Neurodegenerative Disease Management. MSK: consultant at Forum

Pharmaceuticals; editor for Schizophrenia Research. WCL has served on the editorial boards of Epilepsia, Epilepsy \& Behavior and Journal of Neuropsychiatry and Clinical Neurosciences; receives editor's royalties from the publication of Gates and Rowan's Nonepileptic Seizures, 3rd ed. (Cambridge University Press, 2010) and 4th ed. (2017); author's royalties for Taking Control of Your Seizures: Workbook and Therapist Guide (Oxford University Press, 2015); has received research support from the NIH (NINDS 5K23NS45902 [PI]), Rhode Island Hospital, the American Epilepsy Society (AES), the Epilepsy Foundation (EF), Brown University and the Siravo Foundation; serves on the Epilepsy Foundation Professional Advisory Board; has received honoraria for the American Academy of Neurology Annual Meeting Annual Course; has served as a clinic development consultant at University of Colorado Denver, Cleveland Clinic, Spectrum Health and Emory University; and has provided medico legal expert testimony. All other authors report no conflicts of interest.

Patient consent Obtained.

Ethics approval Partners Human Research Committee.

Provenance and peer review Not commissioned; externally peer reviewed.

(c) Article author(s) (or their employer(s) unless otherwise stated in the text of the article) 2017. All rights reserved. No commercial use is permitted unless otherwise expressly granted. 


\section{REFERENCES}

1 Jones B, Reuber M, Norman P. Correlates of health-related quality of life in adults with psychogenic nonepileptic seizures: A systematic review. Epilepsia 2016;57:171-81.

2 Stone J, Warlow C, Sharpe M. The symptom of functional weakness: a controlled study of 107 patients. Brain 2010;133:1537-51.

3 Kranick S, Ekanayake V, Martinez V, et al. Psychopathology and psychogenic movement disorders. Mov Disord 2011;26:1844-50.

4 Perez DL, LaFrance WC. Nonepileptic seizures: an updated review. CNS Spectr 2016;21:239-46.

5 Carson A, Stone J, Hibberd C, et al. Disability, distress and unemployment in neurology outpatients with symptoms 'unexplained by organic disease'. J Neurol Neurosurg Psychiatry 2011;82:810-3.

6 Stone J, Carson A, Duncan R, et al. Symptoms 'unexplained by organic disease' in 1144 new neurology out-patients: how often does the diagnosis change at followup? Brain 2009;132:2878-88.

7 LaFrance WC, Syc S. Depression and symptoms affect quality of life in psychogenic nonepileptic seizures. Neurology 2009;73:366-71.

8 Lawton G, Mayor RJ, Howlett S, et al. Psychogenic nonepileptic seizures and healthrelated quality of life: the relationship with psychological distress and other physical symptoms. Epilepsy Behav 2009;14:167-71.

9 Rawlings GH, Brown I, Reuber M. Predictors of health-related quality of life in patients with epilepsy and psychogenic nonepileptic seizures. Epilepsy Behav 2017;68:153-8.

10 Szaflarski JP, Hughes C, Szaflarski M, et al. Quality of life in psychogenic nonepileptic seizures. Epilepsia 2003;44:236-42.

11 Anderson KE, Gruber-Baldini AL, Vaughan CG, et al. Impact of psychogenic movement disorders versus Parkinson's on disability, quality of life, and psychopathology. Mov Disord 2007;22:2204-9.

12 Pareés I, Saifee TA, Kassavetis $P$, et al. Believing is perceiving: mismatch between selfreport and actigraphy in psychogenic tremor. Brain 2012;135:117-23.

13 Pleizier M, de Haan RJ, Vermeulen M. Management of patients with functional neurological symptoms: a single-centre randomised controlled trial. J Neurol Neurosurg Psychiatry 2017;88:430-6.

14 LaFrance WC, Baird GL, Barry JJ, et al. Multicenter pilot treatment trial for psychogenic nonepileptic seizures: a randomized clinical trial. JAMA Psychiatry 2014;71:997-1005

15 Keshavan MS. Quality of Life as an outcome measure in psychiatric illness. Asian J Psychiatr 2015;18:1.

16 Kanaan RA, Craig TK, Wessely SC, et al. Imaging repressed memories in motor conversion disorder. Psychosom Med 2007:69:202-5

17 Perez DL, Barsky AJ, Daffner K, et al. Motor and somatosensory conversion disorder: a functional unawareness syndrome? J Neuropsychiatry Clin Neurosci 2012;24:141-51.

18 Voon V, Cavanna AE, Coburn K, et al. Functional Neuroanatomy and Neurophysiology of functional neurological disorders (Conversion disorder). J Neuropsychiatry Clin Neurosci 2016;28:168-90.

19 Seeley WW, Menon V, Schatzberg AF, et al. Dissociable intrinsic connectivity networks for salience processing and executive control. J Neurosci 2007;27:2349-56.

20 Linnman C, Moulton EA, Barmettler G, et al. Neuroimaging of the periaqueductal gray: state of the field. Neuroimage 2012;60:505-22.

21 Bickart KC, Hollenbeck MC, Barrett LF, et al. Intrinsic amygdala-cortical functional connectivity predicts social network size in humans. J Neurosci 2012;32:14729-41.

22 Voon V, Brezing C, Gallea C, et al. Emotional stimuli and motor conversion disorder Brain 2010;133:1526-36.

23 Aybek S, Nicholson TR, O'Daly 0, et al. Emotion-motion interactions in conversion disorder: an FMRI study. PLoS One 2015;10:e0123273.

24 Aybek S, Nicholson TR, Zelaya F, et al. Neural correlates of recall of life events in conversion disorder. JAMA Psychiatry 2014;71:52-60.

25 Hassa T, Sebastian A, Liepert J, et al. Symptom-specific amygdala hyperactivity modulates motor control network in conversion disorder. Neuroimage Clin 2017;15:143-50.

26 Stone J, Zeman A, Simonotto E, et al. FMRI in patients with motor conversion symptoms and controls with simulated weakness. Psychosom Med 2007;69:961-9.

27 Voon V, Brezing C, Gallea C, et al. Aberrant supplementary motor complex and limbic activity during motor preparation in motor conversion disorder. Mov Disord 2011;26:2396-403.

28 Labate A, Cerasa A, Mula M, et al. Neuroanatomic correlates of psychogenic nonepileptic seizures: a cortical thickness and VBM study. Epilepsia 2012;53:377-85

29 Ristić AJ, Daković M, Kerr M, et al. Cortical thickness, surface area and folding in patients with psychogenic nonepileptic seizures. Epilepsy Res 2015;112:84-91.

30 Valet M, Gündel H, Sprenger T, et al. Patients with pain disorder show gray-matter loss in pain-processing structures: a voxel-based morphometric study. Psychosom Med 2009;71:49-56.

31 Boeckle M, Liegl G, Jank R, et al. Neural correlates of conversion disorder: overview and meta-analysis of neuroimaging studies on motor conversion disorder. BMC Psychiatry 2016;16:195.

32 Boeckle M, Schrimpf M, Liegl G, et al. Neural correlates of somatoform disorders from a meta-analytic perspective on neuroimaging studies. Neuroimage Clin 2016;11:606-13.
33 Perez DL, Matin N, Barsky A, et al. Cingulo-insular structural alterations associated with psychogenic symptoms, childhood abuse and PTSD in functional neurological disorders. J Neurol Neurosurg Psychiatry 2017;88:491-7.

34 Perez DL, Young SS, King JN, et al. Preliminary predictors of initial attendance, Symptom Burden, and Motor Subtype in a US functional neurological disorders Clinic Population. Cogn Behav Neurol 2016:29:197-205.

35 Matin N, Young SS, Williams B, et al. Neuropsychiatric associations with gender illness Duration, Work Disability, and Motor Subtype in a U.S. functional neurological disorders Clinic Population. J Neuropsychiatry Clin Neurosci 2017:appi.neuropsych. Epub Ahead of Print.

36 Williams DT, Ford B, Fahn S. Phenomenology and psychopathology related to psychogenic movement disorders. Adv Neurol 1995:65:231-57.

37 LaFrance WC, Baker GA, Duncan R, et al. Minimum requirements for the diagnosis of psychogenic nonepileptic seizures: a staged approach: a report from the International League Against Epilepsy Nonepileptic Seizures Task Force. Epilepsia 2013:54:2005-18.

38 American Psychiatric Association. Diagnostic and statistical manual of mental disorders (DSM-5). 5th edition: American Psychiatric Pub, 2013.

39 Ware JE, Sherbourne CD. The MOS 36-item short-form health survey (SF-36). I. Conceptual framework and item selection. Med Care 1992;30:473-83.

40 Spielberger CD, Gorsuch RL, Lushene RE. Manual for the state-trait anxiety inventory: Consulting Psychologists Press, 1970

41 Beck AT, Steer RA, Brown GK. Beck depression inventory-II. San Antonio, TX, 1996:78204-2498

42 Kroenke K, Spitzer RL, Williams JB. The PHQ-15: validity of a new measure for evaluating the severity of somatic symptoms. Psychosom Med 2002;64:258-66

43 Rief W, Hiller W. A new approach to the assessment of the treatment effects of somatoform disorders. Psychosomatics 2003;44:492-8.

44 Rocca MA, Ceccarelli A, Falini A, et al. Brain gray matter changes in migraine patients with T2-visible lesions: a 3-T MRI study. Stroke 2006;37:1765-70.

45 Perez DL, Barsky AJ, Vago DR, et al. A neural circuit framework for somatosensory amplification in somatoform disorders. J Neuropsychiatry Clin NeurosCi 2015;27:e40-e50

46 Perez DL, Dworetzky BA, Dickerson BC, et al. An integrative neurocircuit perspective on psychogenic nonepileptic seizures and functional movement disorders: neural functional unawareness. Clin EEG Neurosci 2015;46:4-15.

47 Craig AD. How do you feel-now? The anterior insula and human awareness. Nat Rev Neurosci 2009:10:59-70.

48 Sridharan D, Levitin DJ, Menon V. A critical role for the right fronto-insular cortex in switching between central-executive and default-mode networks. Proc Natl Acad Sci U S A 2008; 105:12569-74.

49 Nicholson TR, Aybek S, Kempton MJ, et al. A structural MRI study of motor conversion disorder: evidence of reduction in thalamic volume. J Neurol Neurosurg Psychiatry 2014:85:227-9.

50 Aybek S, Nicholson TR, Draganski B, et al. Grey matter changes in motor conversion disorder. J Neurol Neurosurg Psychiatry 2014;85:236-8.

51 Mier D, Bailer J, Ofer J, et al. Neural correlates of an attentional bias to healththreatening stimuli in individuals with pathological health anxiety. J Psychiatry Neurosci 2017; 42:200-9.

52 Etkin A, Klemenhagen KC, Dudman JT, et al. Individual differences in trait anxiety predict the response of the basolateral amygdala to unconsciously processed fearfu faces. Neuron 2004:44:1043-55.

53 van der Plas EA, Boes AD, Wemmie JA, et al. Amygdala volume correlates positively with fearfulness in normal healthy girls. Soc Cogn Affect Neurosci 2010;5:424-31.

54 Baur V, Hänggi J, Jäncke L. Volumetric associations between uncinate fasciculus, amygdala, and trait anxiety. BMC Neurosci 2012;13:4.

55 Holmes AJ, Lee PH, Hollinshead MO, et al. Individual differences in amygdala-media prefrontal anatomy link negative affect, impaired social functioning, and polygenic depression risk. J Neurosci 2012:32:18087-100.

56 Miu AC, Heilman RM, Miclea M. Reduced heart rate variability and vagal tone in anxiety: trait versus state, and the effects of autogenic training. Auton Neurosci 2009;145:99-103.

57 Schlotz W, Schulz P, Hellhammer J, et al. Trait anxiety moderates the impact of performance pressure on salivary cortisol in everyday life. Psychoneuroendocrinology 2006:31:459-72.

58 Bakvis P, Spinhoven P, Putman P, et al. The effect of stress induction on working memory in patients with psychogenic nonepileptic seizures. Epilepsy Behav 2010;19:448-54.

59 Bakvis P, Spinhoven P, Roelofs K. Basal cortisol is positively correlated to threat vigilance in patients with psychogenic nonepileptic seizures. Epilepsy Behav 2009;16:558-60.

60 Reinsberger C, Perez DL, Murphy MM, et al. Pre- and postictal, not ictal, heart rate distinguishes complex partial and psychogenic nonepileptic seizures. Epilepsy Behav 2012:23:68-70.

61 Kozlowska K, Palmer DM, Brown KJ, et al. Reduction of autonomic regulation in children and adolescents with conversion disorders. Psychosom Med 2015;77:356-70 
62 Maurer CW, Liu VD, LaFaver K, et al. Impaired resting vagal tone in patients with functional movement disorders. Parkinsonism Relat Disord 2016;30:18-22.

63 Roelofs K. Freeze for action: neurobiological mechanisms in animal and human freezing. Philos Trans R Soc Lond B Biol Sci 2017;372:20160206.

64 Carrive $P$. The periaqueductal gray and defensive behavior: functional representation and neuronal organization. Behav Brain Res 1993;58:27-47.

65 Canteras NS, Graeff FG. Executive and modulatory neural circuits of defensive reactions: implications for panic disorder. Neurosci Biobehav Rev 2014;46(Pt 3):352-64.

66 Coyle PK, Sterman AB. Focal neurologic symptoms in panic attacks. Am J Psychiatry 1986;143:648-9.

67 Tu CH, Niddam DM, Chao HT, et al. Brain morphological changes associated with cyclic menstrual pain. Pain 2010;150:462-8.

68 Barsky AJ, Goodson JD, Lane RS, et al. The amplification of somatic symptoms. Psychosom Med 1988:50:510-9.

69 Seignourel PJ, Miller K, Kellison I, et al. Abnormal affective startle modulation in individuals with psychogenic [corrected] movement disorder. Mov Disord 2007;22:1265-71

70 Scott DJ, Stohler CS, Egnatuk CM, et al. Placebo and nocebo effects are defined by opposite opioid and dopaminergic responses. Arch Gen Psychiatry 2008:65:220-31.

71 Stoodley CJ, Schmahmann JD. Evidence for topographic organization in the cerebellum of motor control versus cognitive and affective processing. Cortex 2010;46:831-44.

72 Buckner RL, Krienen FM, Castellanos A, et al. The organization of the human cerebellum estimated by intrinsic functional connectivity. J Neurophysiol 2011:106:2322-45
73 Arthuis M, Micoulaud-Franchi JA, Bartolomei F, et al. Resting cortical PET metabolic changes in psychogenic non-epileptic seizures (PNES). J Neurol Neurosurg Psychiatry 2015;86:1106-12.

74 Schrag AE, Mehta AR, Bhatia KP, et al. The functional neuroimaging correlates of psychogenic versus organic dystonia. Brain 2013;136:770-81.

75 Blakemore RL, Sinanaj I, Galli S, et al. Aversive stimuli exacerbate defensive motor behaviour in motor conversion disorder. Neuropsychologia 2016;93:229-41.

76 Wang H, Guo W, Liu F, et al. Clinical significance of increased cerebellar default-mode network connectivity in resting-state patients with drug-naive somatization disorder. Medicine 2016;95:e4043.

77 de Greck M, Scheidt L, Bölter AF, et al. Altered brain activity during emotional empathy in somatoform disorder. Hum Brain Mapp 2012;33:2666-85.

78 Schmahmann JD, Sherman JC. The cerebellar cognitive affective syndrome. Brain 1998;121 (Pt 4):561-79.

79 Moulton EA, Elman I, Pendse G, et al. Aversion-related circuitry in the cerebellum: responses to noxious heat and unpleasant images. J Neurosci 2011;31:3795-804.

80 Kanaan RAA, Duncan R, Goldstein LH, et al. Are psychogenic non-epileptic seizures just another symptom of conversion disorder? I Neurol Neurosurg Psychiatry 2017;88:425-9.

81 Ekanayake V, Kranick S, LaFaver K, et al. Personality traits in psychogenic nonepileptic seizures (PNES) and psychogenic movement disorder (PMD): Neuroticism and perfectionism. J Psychosom Res 2017;97:23-9. Epub ahead of print.

82 Kozlowska K, Griffiths KR, Foster SL, et al. Grey matter abnormalities in children and adolescents with functional neurological symptom disorder. Neuroimage Clin 2017;15:306-14

83 Guo CC, Sturm VE, Zhou J, et al. Dominant hemisphere lateralization of cortical parasympathetic control as revealed by frontotemporal dementia. Proc Natl Acad SCi U SA 2016;113:E2430-E2439. 